\title{
The study of a mixed problem for one class of third order differential equations
}

\author{
Samed J. Aliyev 1*, Arzu G. Aliyeva² and Goncha Z. Abdullayeva
}

\footnotetext{
"Correspondence: samed59@bk.ru ${ }^{1}$ Department of Mechanics and Mathematics, Baku State University, Baku, Azerbaijan

Full list of author information is available at the end of the article
}

\begin{abstract}
This work is dedicated to the study of existence and uniqueness of almost everywhere solution of a multidimensional mixed problem for one class of third order differential equations with nonlinear operator on the right-hand side. The conception of almost everywhere solution for the mixed problem under consideration is introduced. After applying the Fourier method, the solution of the original problem is reduced to the solution of some countable system of nonlinear integro-differential equations in unknown Fourier coefficients of the sought solution. Besides, existence and uniqueness theorems of almost everywhere solution of the mixed problem under consideration are also proved in this work.
\end{abstract}

Keywords: Fourier method; Mixed problem; Almost everywhere solution

\section{Introduction}

This work is dedicated to the study of existence and uniqueness of almost everywhere solution for the following multidimensional mixed problem:

$$
\begin{aligned}
& \frac{\partial^{2} u(t, x)}{\partial t^{2}}-\frac{\partial}{\partial t}(L(u(t, x)))=\Im(u(t, x)) \quad(t \in[0, T], x \in \Omega), \\
& u(0, x)=\varphi(x) \quad(x \in \Omega), \quad u_{t}(0, x)=\psi(x) \quad(x \in \Omega), \\
& \left.u(t, x)\right|_{\Gamma}=0,
\end{aligned}
$$

where $0<T<+\infty ; x=\left(x_{1}, \ldots, x_{n}\right), \Omega$ is a bounded $n$-dimensional domain with an enough smooth boundary $S ; \Gamma=[0, T] \times S$;

$$
L(u(t, x))=\sum_{i, j=1}^{n} \frac{\partial}{\partial x_{i}}\left(a_{i j}(x) \frac{\partial}{\partial x_{j}}\right)-a(x) \cdot u(t, x),
$$

functions $a_{i j}(x)(i, j=\overline{1, n})$ and $a(x)$ are measurable and bounded in $\Omega$ and satisfy in $\Omega$ the following conditions:

$$
a_{i j}(x)=a_{j i}(x), \quad a(x) \geq 0, \quad \sum_{i, j=1}^{n} a_{i j} \xi_{i} \xi_{j} \geq \alpha \cdot \sum_{i=1}^{n} \xi_{i}^{2} \quad(\alpha=\text { const }>0),
$$

(c) The Author(s) 2018. This article is distributed under the terms of the Creative Commons Attribution 4.0 International License (http://creativecommons.org/licenses/by/4.0/), which permits unrestricted use, distribution, and reproduction in any medium, provided you give appropriate credit to the original author(s) and the source, provide a link to the Creative Commons license, and indicate if changes were made. 
where $\xi_{i}(i=1, \ldots, n)$ are arbitrary real numbers; $\varphi, \psi$ are the given functions; $\Im$ is some, generally speaking, nonlinear operator, and $u(t, x)$ is a sought function.

It must be noted that many problems in elasticity theory, in particular the problems of longitudinal vibration of the viscoelastic non-homogeneous bar, some wave problems for elastic-viscidal liquid, etc. lead to the problems of type (1)-(3).

In the beginning, we will note some papers related to problem (1)-(3).

Note that the results of this work is complete progression of the results of [1]. In particular in this work we prove new theorems about existence and uniqueness of the almost everywhere solution of problem (1)-(3).

In [10] a mixed problem for the equation below was considered

$$
\begin{aligned}
& u_{t t}(t, x)-\alpha \Delta u(t, x)-\Delta u_{t}(t, x) \\
& \quad=f\left(t, x, u(t, x), u_{t}(t, x), D u(t, x), D u_{t}(t, x), D^{2} u(t, x), D^{2} u_{t}(t, x)\right) .
\end{aligned}
$$

Under certain special conditions with respect to the nonlinear function $f$ the existence of the solution of the problem under consideration for all $t>0$ has been proved.

In [9], a mixed problem for the equation

$$
u_{t t}(t, x)-\alpha \Delta u_{t}(t, x)-\Delta u(t, x)=f(t, x, u(t, x))
$$

with a nonlinearity of the type $|u|^{p-1}$. $u$ was considered. The conditions for the existence of a global weak solution of this problem are indicated.

Further, in [8] and [2] a special case of problem (1)-(3) is considered, when the operator $\Im$, appearing on the right-hand side of equation (1), is an operator of the type of a function generated by the function $f\left(t, x, u, u_{t}, \nabla u, \nabla u_{t}, \nabla^{2} u\right)$. In [8] by combining the generalized contracted mappings principle and Schauder's fixed point principle for any dimensions $n$, the existence in small theorem (i.e., for sufficiently small values of $T$ ) and the uniqueness in large theorem (i.e., for any finite value of $T$ ) of almost everywhere solution of problem (1)-(3) was proved, and using the method of a priori estimates for all dimensions $n$, the existence in large theorem of almost everywhere solution of problem (1)-(3) was proved. But in [2] the authors investigated the existence in small of classical solution of problem (1)-(3), and using the contracted mappings principle for any dimensions $n$, the existence theorem in small of classical solution of the considered mixed problem was proved.

In the work [7] the existence and uniqueness of the strong global solution of the one special case of problem (1)-(3), when $L=\Delta, n \leq 3$, and $\Im=\Delta u+f(u)$, were proved.

Finally, we mention the work [5] in which theorems about existence and uniqueness of the generalized, almost everywhere, and classical solution for one special one-dimensional case of problem (1)-(3), when $n=1, \Omega=(0,1), L u=\alpha \cdot u_{x x}$, were proved.

\section{Auxiliaries}

In this section, we introduce a number of concepts, notations, and facts to be used later.

1. In investigating the almost everywhere solution of problem (1)-(3), we will use the classes of functions $\stackrel{\circ}{D}$ and $\stackrel{\circ}{D}_{1}$, introduced by Friedrichs [6, p. 38].

We denote by $\dot{D}(\Omega)$ the class of all continuously differentiable functions on $\Omega$ which vanished near the boundary of $\Omega$. We denote the closure of $\dot{D}(\Omega)$ with respect to the norm of $W_{2}^{1}(\Omega)$ by $\stackrel{\circ}{D}(\Omega)$. Hence $\stackrel{\circ}{D}(\Omega) \subset W_{2}^{1}(\Omega)$. 
Denote by $\dot{D_{1}}\left(Q_{T}\right)\left(Q_{T} \equiv[0, T] \times \Omega\right)$ the class of all continuously differentiable functions on the cylinder $Q_{T}$ equal to zero in the $\delta$-neighborhood of the lateral surface on the cylin$\operatorname{der} Q_{T}$, having the form $Q_{T, \delta}=[0, T] \times \Omega_{\delta}$, where $\Omega_{\delta}$ is a $\delta$-neighborhood of the boundary of $\Omega$. We denote the closure of $\dot{D_{1}}\left(Q_{T}\right)$ with respect to the norm of $W_{2}^{1}\left(Q_{T}\right)$ by $\stackrel{\circ}{1}_{1}\left(Q_{T}\right)$. Hence $\stackrel{\circ}{D}_{1}\left(Q_{T}\right) \subset W_{2}^{1}\left(Q_{T}\right)$.

Definition The function $u(t, x) \in \stackrel{\circ}{D}_{1}\left(Q_{T}\right)$, belonging to the space $L_{2}\left(Q_{T}\right)$ together with all its derivatives $u_{t}(t, x), u_{x_{i}}(t, x)(i=\overline{1, n}), u_{t x_{i}}(t, x)(i=\overline{1, n}), u_{x_{i} x_{j}}(t, x)(i, j=\overline{1, n}), u_{t t}(t, x)$, $u_{t x_{i} x_{j}}(t, x)(i, j=\overline{1, n})$, satisfying equation (1) almost everywhere in $Q_{T}$ and taking initial values (2) almost everywhere in $\Omega$, is called an almost everywhere solution of problem (1)-(3).

2. For investigation of problem (1)-(3), we recall one property of the operator $L$, generated by the differential expression (4) and boundary condition (3): there are denumerable number of negative eigenvalues

$$
0>-\lambda_{1}^{2} \geq-\lambda_{2}^{2} \geq \cdots \geq-\lambda_{s}^{2} \geq \cdots \quad\left(0<\lambda_{s} \rightarrow+\infty \text { as } s \rightarrow \infty\right)
$$

with the corresponding generalized eigenfunctions $v_{s}(x)$ which are complete and orthonormal in $L_{2}(\Omega)$. We call the function $v_{s}(x) \in \stackrel{D}{ }(\Omega)$ a generalized eigenfunction of the operator $L$ if it is not identically zero and

$$
\int_{\Omega}\left\{\sum_{i, j=1}^{n} a_{i j}(x) \frac{\partial v_{s}(x)}{\partial x_{i}} \cdot \frac{\partial \Phi(x)}{\partial x_{j}}+a(x) v_{s}(x) \Phi(x)\right\} d x=\lambda_{s}^{2} \cdot \int_{\Omega} v_{s}(x) \Phi(x) d x
$$

for any function $\Phi(x) \in \stackrel{\circ}{D}(\Omega)$.

As the system $\left\{v_{s}(x)\right\}_{s=1}^{\infty}$ is complete orthonormal in $L_{2}(\Omega)$, then it is evident that every almost everywhere solution of problem (1)-(3) has the following form:

$$
u(t, x)=\sum_{s=1}^{\infty} u_{s}(t) v_{s}(x)
$$

where

$$
u_{s}(t)=\int_{\Omega} u(t, x) v_{s}(x) d x \quad(s=1,2, \ldots) .
$$

Then, after applying the Fourier method, finding the unknown Fourier coefficients $u_{s}(t)$ $(s=1,2, \ldots)$, the almost everywhere solution $u(t, x)$ of problem (1)-(3) is reduced to the solution of the following countable system of nonlinear integro-differential equations:

$$
\begin{aligned}
u_{s}(t)= & \varphi_{s}+\frac{1}{\lambda_{s}^{2}}\left(1-e^{-\lambda_{s}^{2} t}\right) \psi_{s} \\
& +\frac{1}{\lambda_{s}^{2}} \int_{0}^{t} \int_{\Omega} \Im(u(\tau, x))\left[1-e^{\lambda_{s}^{2}(t-\tau)}\right] v_{s}(x) d x d \tau \quad(s=1,2, \ldots ; t \in[0, T]),
\end{aligned}
$$

where

$$
\varphi_{s}=\int_{\Omega} \varphi(x) v_{s}(x) d x, \quad \psi_{s}=\int_{\Omega} \psi(x) v_{s}(x) d x \quad(s=1,2, \ldots) .
$$


Proceeding from the definition of almost everywhere solution of problem (1)-(3), it is easy to prove the following.

Lemma If $u(t, x)=\sum_{s=1}^{\infty} u_{s}(t) v_{s}(x)$ is any almost everywhere solution of problem (1)-(3) and the generalized derivatives $\frac{\partial}{\partial x_{k}} a_{i j}(x)(i, j, k=1,2, \ldots, n)$ are bounded on $\Omega$, then functions $u_{s}(t)(s=1,2, \ldots)$ satisfy system $(5)$.

Proof Let $u(t, x)=\sum_{s=1}^{\infty} u_{s}(t) v_{s}(x)$ be any almost everywhere solution of problem (1)-(3). Then it is evident that

$$
\int_{0}^{T} \int_{\Omega}\left\{u_{t t}(t, x)-\frac{\partial}{\partial t}(L(u(t, x)))-\Im(u(t, x))\right\} \Phi(t, x) d x d t=0
$$

for each $\Phi(t, x) \in L_{2}\left(Q_{T}\right)$.

If, in particular, we take

$$
\Phi(t, x)= \begin{cases}(t-\tau)^{2} v_{s}(x) & \text { for } 0 \leq t \leq \tau, x \in \Omega \\ 0 & \text { for } \tau<t \leq T, x \in \Omega\end{cases}
$$

where $s=1,2, \ldots$ and $\tau \in[0, T]$, then with the help of integration by parts with respect to $t$ twice in the first term and once in the second term of (6) and taking the initial conditions (2) into consideration, we easily get

$$
\begin{aligned}
& 2 \int_{0}^{\tau} u_{s}(t) d t-2 \lambda_{s}^{2} \int_{0}^{\tau}(t-\tau) u_{s}(t) d t-\int_{0}^{\tau}(t-\tau)^{2} \Im_{s}(u, t) d t \\
& \quad-2 \tau \varphi_{s}-\tau^{2} \psi_{s}-\lambda_{s}^{2} \tau^{2} \varphi_{s}=0
\end{aligned}
$$

where

$$
\Im_{s}(u, t) \equiv \int_{\Omega} \Im(u(t, x)) v_{s}(x) d x .
$$

Differentiating (7) three times with respect to $\tau$, we have the next problem

$$
\left\{\begin{array}{l}
u_{s}^{\prime \prime}(\tau)+\lambda_{s}^{2} u_{s}^{\prime}(\tau)=\Im_{s}(u, \tau) \quad(s=1,2, \ldots ; \tau \in[0, T]), \\
u_{s}(0)=\varphi_{s}, \quad u_{s}^{\prime}(0)=\psi_{s}
\end{array}\right.
$$

which is obviously equivalent to system (5). The lemma is proved.

3. We denote by $B_{\beta_{0}, \ldots, \beta_{l}, T}^{\alpha_{0}, \ldots, \alpha_{l}}$ a totality of all the functions of the form

$$
u(t, x)=\sum_{s=1}^{\infty} u_{s}(t) v_{s}(x)
$$

considered in $Q_{T}=[0, T] \times \Omega$, where $u_{s}(t) \in C^{(l)}([0, T])$ for all $s$ and

$$
N_{T}(u) \equiv \sum_{i=0}^{l}\left\{\sum_{s=1}^{\infty}\left(\lambda_{s}^{\alpha_{i}} \cdot \max _{0 \leq t \leq T}\left|u_{s}^{(i)}(t)\right|\right)^{\beta_{i}}\right\}^{\frac{1}{\beta_{i}}}<+\infty,
$$


with $\alpha_{i} \geq 0,1 \leq \beta_{i} \leq 2(i=0,1, \ldots, n)$. We define the norm in this set as $\|u\|=N_{T}(u)$. It is evident that all these spaces are Banach spaces [4, p. 50].

4. Let $G$ be a class all functions $u(t, x)$ which have the properties $u(t, x), u_{t}(t, x), u_{x_{i}}(t, x)$ $(i=\overline{1, n}), u_{t x_{i}}(t, x)(i=\overline{1, n}), u_{x_{i} x_{j}}(t, x)(i, j=\overline{1, n}), u_{t t}(t, x), u_{t x_{i} x j}(t, x)(i, j=\overline{1, n}) \in L_{2}\left(Q_{T}\right)$.

5. We agree to assume that all the quantities throughout this work are real, all the functions are real-valued, and all the integrals are understood in the sense of Lebesgue.

\section{On the existence of almost everywhere solution}

In this section, using Zabreyko and Krasnoselskiy's fixed point principle, the following existence theorem for the almost everywhere solution of problem (1)-(3) is proved for $n$.

\section{Theorem 1 Let:}

1. $a_{i j}(x) \in C^{(2)}(\bar{\Omega})(i, j=\overline{1, n}) ; a(x) \in C^{(1)}(\bar{\Omega}) ; S \in C^{(3)}$; the eigenfunctions $v_{s}(x)$ of the operator $L$ under boundary condition $\left.v_{s}(x)\right|_{S}=0$ be three times continuously differentiable on $\bar{\Omega} ; \varphi(x) \in W_{2}^{3}(\Omega), \varphi(x), L \varphi(x) \in \stackrel{\circ}{D}(\Omega) ; \psi(x) \in W_{2}^{2}(\Omega) \cap \stackrel{\circ}{D}(\Omega)$.

2. $\Im=\Im_{1}+\Im_{2}$, where

(a) the operator $\Im_{1}$ acts from the closed ball $K_{1}\left(\|u\|_{B_{2, T}^{2}} \leq \frac{1}{\lambda_{1}} R\right)$ into the space $W_{t, x, 2}^{0,1}\left(Q_{T}\right)$ continuously, where $R>\|W(t, x)\|_{B_{2,2, T}^{3,2}}$ and

$$
W(t, x)=\sum_{s=1}^{\infty}\left\{\varphi_{s}+\frac{1}{\lambda_{s}^{2}}\left[1-e^{-\lambda_{s}^{2} t}\right] \psi_{s}\right\} \cdot v_{s}(x) ;
$$

(b) the operator $\Im_{2}$ acts from the closed ball $K_{2}\left(\|u\|_{B_{2,2, T}^{3,2}} \leq R\right)$ into the space $W_{t, x, 2}^{0,1}\left(Q_{T}\right)$ and satisfies the Lipschitz condition on this closed ball:

$$
\left\|\Im_{2}(u)-\Im_{2}(v)\right\|_{W_{t,, 2,2}^{0,1}\left(Q_{T}\right)} \leq q \cdot\|u-v\|_{B_{2,2, T}^{3,2}}
$$

where

$$
\begin{aligned}
& \left(\sqrt{T}+\frac{1}{\sqrt{2}}\right) \cdot C_{0} \cdot q \equiv q_{0}<1, \\
& C_{0} \equiv \max \left\{n \cdot \max _{i, j=1, n}\left\{\left\|a_{i j}(x)\right\|_{C(\bar{\Omega})}\right\},\|a(x)\|_{C(\bar{\Omega})}\right\}^{\frac{1}{2}} .
\end{aligned}
$$

3.

$$
\|W(t, x)\|_{B_{2,2, T}^{3,2}}+\left(\sqrt{T}+\frac{1}{\sqrt{2}}\right) \cdot C_{0} \cdot \sup _{u \in K_{2}}\left\{\|\Im(u)\|_{W_{t, x, 2}^{0,1}\left(Q_{T}\right)}\right\} \leq R
$$

4. For any $u \in K_{2}$, for almost all $t \in[0, T]$,

$$
\Im_{i}(u(t, x)) \in \stackrel{\circ}{D}(\Omega), \quad i=1,2 .
$$

Then problem (1)-(3) has an almost everywhere solution.

Proof From condition 1 of the theorem it follows that $W(t, x) \in B_{2,2, T}^{3,2}$ since $\sum_{s=1}^{\infty}\left(\lambda_{s}^{3} \varphi_{s}\right)^{2}<$ $+\infty, \sum_{s=1}^{\infty}\left(\lambda_{s} \psi_{s}\right)^{2}<+\infty$ and $\sum_{s=1}^{\infty}\left(\lambda_{s}^{2} \psi_{s}\right)^{2}<+\infty$. 
We consider the operators $Q_{1}(u)=W+P \Im_{1}(u)$ and $Q_{2}(u)=P \Im_{2}(u)$ in the closed ball $K_{2}\left(\|u\|_{B_{2,2, T}^{3,2}} \leq R\right)$, where the function $W(t, x)$ is defined by $(8)$ and

$$
P(u(t, x)) \equiv \sum_{s=1}^{\infty} \frac{1}{\lambda_{s}^{2}} \int_{0}^{t} \int_{\Omega} u(\tau, \xi) v_{s}(\xi) \cdot\left[1-e^{-\lambda_{s}^{2}(t-\tau)}\right] d \xi d \tau \cdot v_{s}(x)
$$

Using condition 4 of this theorem and the relation $L v_{s}(x)=-\lambda_{s}^{2} v_{s}(x)$, we transform (by integrating by parts) system (5) to the following form:

$$
\begin{aligned}
u_{s}(t)= & \varphi_{s}+\frac{1}{\lambda_{s}^{2}}\left(1-e^{-\lambda_{s}^{2} t}\right) \psi_{s}+\frac{1}{\lambda_{s}^{3}} \int_{0}^{t} \int_{\Omega}\left[\sum_{i, j=1}^{n} a_{i j}(x) \frac{\partial}{\partial x_{i}} \Im(u(\tau, x)) \cdot \frac{\partial}{\partial x_{j}}\left(\frac{v_{s}(x)}{\lambda_{s}}\right)\right. \\
& \left.+a(x) \Im(u(\tau, x)) \cdot \frac{v_{s}(x)}{\lambda_{s}}\right] \cdot\left[1-e^{-\lambda_{s}^{2}(t-\tau)}\right] d x d \tau, \quad s=1,2, \ldots ; t \in[0, T] .
\end{aligned}
$$

Then we have, for every $u \in K_{2}$,

$$
\begin{aligned}
Q_{1}(u(t, x))= & W(t, x)+\sum_{s=1}^{\infty} \frac{1}{\lambda_{s}^{3}} \int_{0}^{t} \int_{\Omega}\left[\sum_{i, j=1}^{n} a_{i j}(\xi) \frac{\partial}{\partial \xi_{i}} \Im_{1}(u(\tau, \xi)) \cdot \frac{\partial}{\partial \xi_{j}}\left(\frac{v_{s}(\xi)}{\lambda_{s}}\right)\right. \\
& \left.+a(\xi) \Im_{1}(u(\tau, \xi)) \cdot \frac{v_{s}(\xi)}{\lambda_{s}}\right] \cdot\left[1-e^{-\lambda_{s}^{2}(t-\tau)}\right] d \xi d \tau \cdot v_{s}(x), \\
Q_{2}(u(t, x))= & \sum_{s=1}^{\infty} \frac{1}{\lambda_{s}^{3}} \int_{0}^{t} \int_{\Omega}\left[\sum_{i, j=1}^{n} a_{i j}(\xi) \frac{\partial}{\partial \xi_{i}} \Im_{2}(u(\tau, \xi)) \cdot \frac{\partial}{\partial \xi_{j}}\left(\frac{v_{s}(\xi)}{\lambda_{s}}\right)\right. \\
& \left.+a(\xi) \Im_{2}(u(\tau, \xi)) \cdot \frac{v_{s}(\xi)}{\lambda_{s}}\right] \cdot\left[1-e^{-\lambda_{s}^{2}(t-\tau)}\right] d \xi d \tau \cdot v_{s}(x) .
\end{aligned}
$$

It is easy to obtain that, for any $u, v \in K_{2}$,

$$
\begin{aligned}
\left\|Q_{1}(u)+Q_{2}(u)\right\|_{B_{2,2, T}^{3,2}} \\
=\left\|W(t, x)+P \Im_{1}(u(t, x))+P \Im_{2}(u(t, x))\right\|_{B_{2,2, T}^{3,2}} \\
\leq\|W(t, x)\|_{B_{2,2, T}^{3,2}}+\left\|P \Im_{1}(u(t, x))+P \Im_{2}(u(t, x))\right\|_{B_{2,2, T}^{3,2}} \\
\leq\|W(t, x)\|_{B_{2,2, T}^{3,2}}+\left(\sqrt{T}+\frac{1}{\sqrt{2}}\right) \\
\quad \cdot\left\{\int_{0}^{T} \int_{\Omega}\left[\sum_{i, j=1}^{n} a_{i j}(\xi) \frac{\partial \Im(u(\tau, \xi))}{\partial \xi_{i}} \cdot \frac{\partial \Im(u(\tau, \xi))}{\partial \xi_{j}}+a(\xi)(\Im(u(\tau, \xi)))^{2}\right] d \xi d \tau\right\}^{\frac{1}{2}} \\
\leq\|W(t, x)\|_{B_{2,2, T}^{3,2}}+\left(\sqrt{T}+\frac{1}{\sqrt{2}}\right) \cdot C_{0}\|\Im(u(t, x))\|_{W_{t, x, 2}^{0,1}\left(Q_{T}\right)} \leq R, \\
\left\|Q_{1}(u)-Q_{1}(v)\right\|_{B_{2,2, T}^{3,2}} \\
\leq\left(\sqrt{T}+\frac{1}{\sqrt{2}}\right) \cdot\left\{\int _ { 0 } ^ { T } \int _ { \Omega } \left[\sum_{i, j=1}^{n} a_{i j}(\xi) \frac{\partial}{\partial \xi_{i}}\left(\Im_{1}(u(\tau, \xi))-\Im_{1}(v(\tau, \xi))\right)\right.\right.
\end{aligned}
$$




$$
\begin{aligned}
& \left.\left.\quad \times \frac{\partial}{\partial \xi_{j}}\left(\Im_{1}(u(\tau, \xi))-\Im_{1}(v(\tau, \xi))\right)+a(\xi)\left(\Im_{1}(u(\tau, \xi))-\Im_{1}(v(\tau, \xi))\right)^{2}\right] d \xi d \tau\right\}^{\frac{1}{2}} \\
& \leq\left(\sqrt{T}+\frac{1}{\sqrt{2}}\right) \cdot C_{0}\left\|\Im_{1}(u(t, x))-\Im_{1}(v(t, x))\right\|_{W_{t, x, 2}^{0,1}\left(Q_{T}\right)} \\
& \left\|Q_{2}(u)-Q_{2}(v)\right\|_{B_{2,2, T}^{3,2}} \\
& \leq\left(\sqrt{T}+\frac{1}{\sqrt{2}}\right) \cdot C_{0}\left\|\Im_{2}(u(t, x))-\Im_{2}(v(t, x))\right\|_{W_{t, x, 2}^{0,1}\left(Q_{T}\right)} \\
& \leq\left(\sqrt{T}+\frac{1}{\sqrt{2}}\right) \cdot C_{0} \cdot q \cdot\|u-v\|_{B_{2,2, T}^{3,2}}=q_{0}\|u-v\|_{B_{2,2, T}, 2}
\end{aligned}
$$

where $\Im(u(t, x))=\Im_{1}(u(t, x))+\Im_{2}(u(t, x))$ and the number $C_{0}$ is defined by $(10)$.

From inequality (15), which holds not only for $u, v \in K_{2}$, but also for all $u, v \in K_{1}$, follows that the operator $Q_{1}$ acts continuously from the closed ball $K_{1}$ into $B_{2,2, T}^{3,2}$. Since the closed ball $K_{2}$ is imbedded into the closed ball $K_{1}$ compactly by virtue of [4, Theorem 1.1, p. 51], then the operator $Q_{1}$ acts compactly from $K_{2}$ into $B_{2,2, T}^{3,2}$. Further, since $q_{0}<1$, then from inequality (16) it is seen that the operator satisfies a Lipschitz condition on $K_{2}$ with the coefficient which is less than of unity. From inequality (14) it is seen that the operator $Q=Q_{1}+Q_{2}$ acts from the closed ball $K_{2}$ into itself. Hence, by virtue of the principle of Zabreyko and Krasnoselskiy about fixed point [11, p. 1234], the operator $Q$ has at least one fixed point $u(x, t)$ in the closed ball $K_{2}$, which is easy to verify (in absolutely the same way as in the proof of Theorem of [1]), which is an almost everywhere solution of problem (1)-(3). The theorem is proved.

\section{On the existence and uniqueness of almost everywhere solution}

In this section, using the successive approximations method, the following existence and uniqueness theorem for the almost everywhere solution of problem (1)-(3) is proved for $n$.

\section{Theorem 2 Let:}

1. Condition 1 of Theorem 1 be satisfied.

2. The operator $\Im$ act from $B_{2,2, T}^{3,2} \cup\left(G \cap B_{2,2, T}^{2,1}\right)$ into $W_{t, x, 2}^{0,1}\left(Q_{T}\right)$ so that, for all $u \in B_{2,2, T}^{3,2}$ and $t \in[0, T]$,

$$
\|\Im(u(t, x))\|_{W_{2}^{1}(\Omega)} \leq a(t)+b(t) \cdot\|u\|_{B_{2,2, T}^{3,2}}
$$

where $a(t), b(t) \in L_{2}(0, T)$.

3. For each $u \in B_{2,2, T}^{3,2} \cup\left(G \cap B_{2,2, T}^{2,2}\right)$ for almost all $t \in[0, T] \Im(u(t, x)) \in \stackrel{D}{(\Omega)}$.

4. For any $u, v \in K_{0}\left(\|u\|_{B_{2,2, T}^{3,2}} \leq a_{0}\right)$ and $t \in[0, T]$,

$$
\|\Im(u(t, x))-\Im(v(t, x))\|_{W_{2}^{1}(\Omega)} \leq C(t) \cdot\|u-v\|_{B_{2,2, T}^{3,2}},
$$

where $c(t) \in L_{2}(0, T)$,

$$
\begin{aligned}
a_{0} \equiv & \left\{\left[2\|W(t, x)\|_{B_{2,2, T}^{3,2}}^{2}+4(2 T+1) \cdot C_{0}^{2} \cdot\|a(t)\|_{L_{2}(0, T)}^{2}\right]\right. \\
& \left.\cdot \exp \left[4(2 T+1) \cdot C_{0}^{2} \cdot\|b(t)\|_{L_{2}(0, T)}^{2}\right]\right\}^{\frac{1}{2}}
\end{aligned}
$$

the function $W(t, x)$ is defined by (8) and the number $C_{0}$ is defined by (10). 
Then problem (1)-(3) has a unique almost everywhere solution $u(t, x)$ in $B_{2,2, T}^{3,2}$, which can be found by the method of successive approximation. Moreover, the distance between the solution $u(t, x)$ and the kth approximation $u_{k}(t, x)$ is estimated by the following formula:

$$
\left\|u_{k}(t, x)-u(t, x)\right\|_{B_{2,2, T}^{3,2}} \leq a_{0} \cdot \frac{\left\{\sqrt{2 T+1} \cdot C_{0} \cdot\|C(t)\|_{L_{2}(0, T)}\right\}^{k}}{\sqrt{k !}} \quad(k=0,1,2, \ldots) .
$$

Proof We consider the following operator $Q$ :

$$
Q(u(t, x))=W(t, x)+P \Im(u(t, x))
$$

where the function $W(t, x)$ is defined by $(8)$ and

$$
P \Im(u(t, x)) \equiv \sum_{s=1}^{\infty} \frac{1}{\lambda_{s}^{2}} \int_{0}^{t} \int_{\Omega} \Im(u(\tau, \xi)) \cdot v_{s}(\xi)\left[1-e^{-\lambda_{s}^{2}(t-\tau)}\right] d \xi d \tau \cdot v_{s}(x) .
$$

Then it is obvious that the operator $Q$ acts in the space $B_{2,2, T}^{3,2}$ and satisfies the condition

$$
\|Q(u)-Q(v)\|_{B_{2,2, T}^{3,2}} \leq \sqrt{2 T+1} \cdot C_{0} \cdot\|C(t)\|_{L_{2}(0, T)} \cdot\|u-v\|_{B_{2,2, T}^{3,2}}
$$

in the ball $K_{0}$, where $C_{0}$ is defined by (10).

Consider in $B_{2,2, T}^{3,2}$ the sequence $u_{k}(t, x)=Q\left(u_{k-1}(t, x)\right)$, where $u_{0}(t, x) \equiv 0$ in $Q_{T}$. Using inequality (17) and applying the inductions method, it is easy to have, for any $k(k=1,2, \ldots)$ and $t \in[0, T]$,

$$
\begin{aligned}
\left\|u_{k}\right\|_{B_{2,2, T}}^{2,2} & =\left\|Q\left(u_{k-1}\right)\right\|_{B_{2,2, T}^{3,2}}^{2}=\left\|W+P \Im\left(u_{k-1}(t, x)\right)\right\|_{B_{2,2, T}^{3,2}}^{2} \\
& \leq 2\|W\|_{B_{2,2, T}^{3,2}}^{2}+2\left\|P \Im\left(u_{k-1}(t, x)\right)\right\|_{B_{2,2, T}^{3,2}}^{2} \\
& \leq 2\|W\|_{B_{2,2, T}^{3,2}}^{2}+2(2 T+1) \cdot C_{0}^{2} \cdot \int_{0}^{t}\left\|\Im\left(u_{k-1}(\tau, x)\right)\right\|_{W_{2}^{1}(\Omega)}^{2} d \tau \\
& \leq 2\|W\|_{B_{2,2, T}^{3,2}}^{2}+4(2 T+1) \cdot C_{0}^{2} \cdot \int_{0}^{t}\left\{a^{2}(\tau)+b^{2}(\tau) \cdot\left\|u_{k-1}\right\|_{B_{2,2, T}^{3,2}}^{2}\right\} d \tau \\
& \leq A^{2}+A^{2} \cdot \int_{0}^{t} B^{2}(\tau) d \tau+\cdots+A^{2} \frac{\left\{\int_{0}^{t} B^{2}(\tau) d \tau\right\}^{k}}{k !},
\end{aligned}
$$

where

$$
\begin{aligned}
& A^{2} \equiv 2\|W\|_{B_{2,2, T}^{3,2}}^{2}+4(2 T+1) \cdot C_{0}^{2} \cdot\|a(t)\|_{L_{2}(0, T)}^{2}, \\
& B^{2}(t) \equiv 4(2 T+1) \cdot C_{0}^{2} \cdot b^{2}(t) .
\end{aligned}
$$

From (23) it follows that, for any $k(k=0,1,2, \ldots)$,

$$
\left\|u_{k}\right\|_{B_{2,2, T}^{3,2}}^{2} \leq A^{2} \exp \left\{\int_{0}^{T} B^{2}(t) d t\right\} \equiv a_{0}^{2},
$$

that is, all the approximations $u_{k}(t, x)$ are contained in the ball $K_{0}$. 
Next, using condition 4 of this theorem and taking (24) into account, we have, for every $k(k=1,2, \ldots)$ and $t \in[0, T]$,

$$
\begin{aligned}
\left\|u_{k+1}-u_{k}\right\|_{B_{2,2, T}^{3,2}}^{2} & =\left\|P \Im\left(u_{k}(t, x)\right)-P \Im\left(u_{k-1}(t, x)\right)\right\|_{B_{2,2, T}^{3,2}}^{2} \\
& \leq(2 T+1) \cdot C_{0}^{2} \cdot \int_{0}^{t}\left\|\Im\left(u_{k}(\tau, x)\right)-\Im\left(u_{k-1}(\tau, x)\right)\right\|_{W_{2}^{1}(\Omega)}^{2} d \tau \\
& \leq(2 T+1) \cdot C_{0}^{2} \cdot \int_{0}^{t} C^{2}(\tau) \cdot\left\|u_{k}-u_{k-1}\right\|_{B_{2,2, \tau}^{3,2}}^{2} d \tau \\
& \leq\left\|u_{1}-u_{0}\right\|_{B_{2,2, \tau}^{3,2}}^{2} \cdot \frac{\left\{(2 T+1) \cdot C_{0}^{2} \int_{0}^{t} C^{2}(\tau) d \tau\right\}^{k}}{k !} \\
& =\left\|u_{1}\right\|_{B_{2,2, T}^{3,2}}^{2} \cdot \frac{\left\{(2 T+1) \cdot C_{0}^{2} \int_{0}^{t} C^{2}(\tau) d \tau\right\}^{k}}{k !} .
\end{aligned}
$$

Consequently,

$$
\left\|u_{k+1}(t, x)-u_{k}(t, x)\right\|_{B_{2,2, T}^{3,2}}^{2} \leq a_{0}^{2} \cdot \frac{\left\{(2 T+1) \cdot C_{0}^{2}\|c(t)\|_{L_{2}(0, T)}^{2}\right\}^{k}}{k !} \quad(k=0,1,2, \ldots) .
$$

From this it follows that the sequence $\left\{u_{k}(t, x)\right\}_{k=1}^{\infty}$ forms the Cauchy sequence in $B_{2,2, T}^{3,2}$. Due to the completeness of the space $B_{2,2, T}^{3,2}$, we have

$$
u_{k}(t, x) \stackrel{B_{2,2, T}^{3,2}}{\rightarrow} u(t, x) \in K_{0} \quad \text { as } k \rightarrow \infty
$$

Since the operator $Q$ is continuous in the ball $K_{0}$ (see inequality (22)), from the relations $u_{k}(t, x)=Q\left(u_{k-1}(t, x)\right)$ it follows that

$$
u(t, x)=Q(u(t, x))
$$

It is easy to verify (in absolutely the same way as in the proof of Theorem of [1]) that the function $u(t, x)$ is the almost everywhere solution of problem (1)-(3). Inequality (20) can be proved similarly to (25) and (26).

Now, we prove the uniqueness of the almost everywhere solution of problem (1)-(3) in $B_{2,2, T}^{3,2}$. Let $u(t, x)=\sum_{s=1}^{\infty} u_{s}(t) v_{s}(x)$ be any almost everywhere solution of problem (1)-(3) in $B_{2,2, T}^{3,2}$. Then $\Im(u(t, x)) \in L_{2}\left(Q_{T}\right)$. By virtue of the lemma in Sect. 2 , the functions $u_{s}(t)$ $(s=1,2, \ldots)$ satisfy system (5). From system (5) we obtain

$$
\|u(t, x)\|_{B_{2,2, T}^{2,1}} \leq\|W(t, x)\|_{B_{2,2, T}^{2,1}}+\left(\sqrt{T}+\frac{1}{\sqrt{2}}\right) \cdot\|\Im(u(t, x))\|_{L_{2}\left(Q_{T}\right)}<+\infty .
$$

Consequently, $u(t, x) \in B_{2,2, T}^{2,1}$. As $u(t, x) \in G \cap B_{2,2, T}^{2,1}$, then due to condition 2 of this theorem $\Im(u(t, x)) \in W_{t, x, 2}^{0,1}\left(Q_{T}\right)$, and due to condition 3 of this theorem for almost all $t \in[0, T]$ $\Im(u(t, x)) \in \stackrel{\circ}{ }(\Omega)$. Using this, from system (13) equivalent to system (5), we have

$$
\|u(t, x)\|_{B_{2,2, T}^{3,2}} \leq\|W(t, x)\|_{B_{2,2, T}^{3,2}}+\left(\sqrt{T}+\frac{1}{\sqrt{2}}\right) \cdot C_{0}\|\Im(u(t, x))\|_{W_{t, x, 2}^{0,1}\left(Q_{T}\right)}<+\infty .
$$


Consequently, $u(t, x) \in B_{2,2, T}^{3,2}$. Then, using inequality (17), similar to (23) and (24), $\forall t \in$ $[0, T]$ we have

$$
\|u\|_{B_{2,2, T}^{3,2}}^{2} \leq a_{0}^{2}
$$

Thus, all the possible almost everywhere solutions of problem (1)-(3) belong to the sphere $K_{0}$, and there is a fixed point in $B_{2,2, T}^{3,2}$ of the operator $Q$ defined by $(21)$. Let $u(t, x)$ and $v(t, x)$ be two arbitrary almost everywhere solutions of problem (1)-(3). Then, using the fact that $u(t, x), v(t, x) \in K_{0}$ and condition 4 of the present theorem, it is easy to obtain that, $\forall t \in[0, T]$,

$$
\begin{aligned}
\|u-v\|_{B_{2,2, t}^{3,2}}^{2} & \leq(2 T+1) C_{0}^{2} \cdot \int_{0}^{t}\|\mathfrak{s}(u(\tau, x))-\Im(v(\tau, x))\|_{W_{2}^{1}(\Omega)}^{2} d \tau \\
& \leq(2 T+1) \cdot C_{0}^{2} \cdot \int_{0}^{t} c^{2}(\tau) \cdot\|u-v\|_{B_{2,2, \tau}^{3,2}}^{2} d \tau .
\end{aligned}
$$

From here, on applying Bellman's inequality [3, pp. 188, 189], we obtain that $\forall t \in[0, T]$ $\|u-v\|_{B_{2,2, T}^{3,2}} \equiv 0$. Hence, $u=v$. The theorem is proved.

\section{Acknowledgements}

Not applicable.

Funding

Not applicable.

Competing interests

The authors declare that they have no competing interests.

Authors' contributions

All authors conceived of the study, participated in its design and coordination, read and approved the final manuscript.

\section{Author details}

'Department of Mechanics and Mathematics, Baku State University, Baku, Azerbaijan. ${ }^{2}$ Department of Differential Equations, Institute of Mathematics and Mechanics, Azerbaijan National Academy of Sciences, Baku, Azerbaijan.

\section{Publisher's Note}

Springer Nature remains neutral with regard to jurisdictional claims in published maps and institutional affiliations.

Received: 23 February 2018 Accepted: 7 June 2018 Published online: 15 June 2018

\section{References}

1. Aliyev, S., Aliyeva, A.: On the existence for almost everywhere solution of multidimensional mixed problem for one class third order differential equations with nonlinear operator in the right-hand side. Int. J. Pure Appl. Math. 115(3), 549-560 (2017)

2. Aliyev, S., Aliyeva, A.: The study of multidimensional mixed problem for one class of third order semilinear pseudohyperbolic equations. Eur. J. Pure Appl. Math. 10(5), 1078-1091 (2017)

3. Beckenbach, E., Bellman, R.: Inequalities. Mir, Moscow (1965) (in Russian)

4. Khudaverdiyev, K.I.: Multidimensional Mixed Problem for Nonlinear Hyperbolic Equations. Az. Gostekn. University Publ., Baku (2011) (in Russian)

5. Khudaverdiyev, K.I., Veliyev, A.A.: Study of the One Dimensional Mixed Problem for a Class of Third Order Pseudohyperbolic Equations with Nonlinear Operator Right Side. Chashioglu, Baku (2010) (in Russian)

6. Ladyzhenskaya, O.A.: Mixed Problem for Hyperbolic Equations. Gostekhizdat, Moscow (1953) (in Russian)

7. Webb, G.F.: Existence and asymptotic behavior for a strongly damped nonlinear wave equation. Can. J. Math. 3, 631-643 (1980)

8. Xudaverdiyev, K.I., Aliyev, S.: A Multidimensional Mixed Problem for One Class of Semilinear Pseudohyperbolic Equations of the Third Order. Az. Gostekn. University Publ., Baku (2012) (in Russian)

9. Yu, T., Yang, H.-O.: Initial boundary value problem for a class of strongly damped nonlinear wave equation. J. Harbin Eng. Univ. 25(2), 254-256 (2004)

10. Yukiyoshi, E.: On some nonlinear evolution equations with the strong dissipation, III. J. Differ. Equ. 45(3), 332-355 (1982)

11. Zabreyko, P.P., Krasnoselskiy, M.A.: One technique for getting a new fixed-point principles. Dokl. Akad. Nauk SSSR 176(6), 1233-1235 (1967) (in Russian) 